\title{
An Approach to Modeling the Impact of Traffic Incident on Urban Expressway
}

\author{
Yaping Li, ${ }^{1,2}$ Jian Lu, ${ }^{1,2}$ HongWu Li, ${ }^{3}$ Huihui Xiao, ${ }^{1,2}$ and Qingchao Liu ${ }^{1,2}$ \\ ${ }^{1}$ Southeast University Key Laboratory of Urban ITS of Jiangsu Province, No. 2 Sipailou, Nanjing 210096, China \\ ${ }^{2}$ Jiangsu Province Collaborative Innovation Center of Modern Urban Traffic Technologies, No. 2 Sipailou, Nanjing 210096, China \\ ${ }^{3}$ Traffic Police Corps of Jiangsu Provincial Public Security Bureau, No. 1 Yangzhou Road, Nanjing 210024, China
}

Correspondence should be addressed to Jian Lu; lujian_1972@seu.edu.cn

Received 17 May 2015; Revised 7 July 2015; Accepted 8 July 2015

Academic Editor: Gabriella Bretti

Copyright (C) 2015 Yaping Li et al. This is an open access article distributed under the Creative Commons Attribution License, which permits unrestricted use, distribution, and reproduction in any medium, provided the original work is properly cited.

\begin{abstract}
To identify network bottlenecks of urban expressway effectively is a foundational work for improving network traffic condition and preventing traffic congestion. This study proposes a methodology to estimate the impact of traffic incident on urban expressway on the basis of modified cell transmission model. The metastable state was taken into account in the proposed method to reflect the actual operating state of traffic flow on urban expressway as much as possible. Regarding the location of traffic incident, the method of cell restructuring settings was discussed. We then proceed to introduce a new concept of the effected length in a given time period as the evaluation indicator to directly depict the influence of traffic incident. The proposed method was tested on a 6516-meter urban expressway section of west second ring road in Beijing. The simulation results indicated that the proposed methodology performs well to predict the impact of traffic incident on urban expressway.
\end{abstract}

\section{Introduction}

Traffic incident has become one of the main causes of urban expressway congestion. Travel time delays occur due to lane blockages following incident and the corresponding expressway capacity reduction. From this perspective, identifying the influence scope of traffic incident on urban expressway would be of great interest in traffic and vehicle management strategies in real time. It is thus necessary to develop a proper methodology which is able to estimate the impact of traffic incident on urban expressway.

A number of existing studies have attempted to identify the impact of traffic incident on traffic network. Wright and Roberg [1] established a simple model for jam growth arising from a single bottleneck of an idealized grid network. Skabardonis et al. [2] utilized the loop detectors data to calculate the average and the probability distribution of delays. Kwon and Varaiya [3] presented a method, which can measure the delay that collisions cause, the potential reduction in delay at bottlenecks that ideal ramp metering can achieve, and the remaining delay in any freeway section. Long et al. [4] developed a congestion propagation model of urban network traffic based on the cell transmission model. Smid [5] proposed a methodology operationalized in a model, which is able to predict congestion on motorways without knowledge of the actual traffic conditions. In existing literatures, some traffic flow models were adopted to study the traffic flow variation in time and space, such as traffic wave model, probabilistic model, car-following model, and cell transmission model (CET). Compared with other models, CET put forward by Daganzo $[6,7]$ is found to be an effective tool in dynamic simulation of traffic flow because of availability in different traffic flow conditions and higher computational efficiency. Muñoz et al. [8] presented a semiautomated method for calibrating the parameters of a modified version of CET and applied to loop detector data to determine freeflow speeds, congestion-wave speeds, and jam densities for specified subsections of a freeway segment. Szeto et al. [9] developed a short-term space-time traffic flow forecasting strategy integrating the empirical-based seasonal autoregressive integrated moving average (SARIMA) time-series forecasting technique with the CET. Long et al. [10] used the CET and apply it to simulate the formation and dissipation of traffic jams at the microscopic level. Sumalee et al. [11] 
modeled traffic flow density on freeway segments with stochastic demand and supply on the basis of CTM. Generally, the research object of existing studies, which studied the effect of traffic incident, is freeway. And the underlying assumption of these existing models is the relationship between traffic flow and density which is triangular fundamental diagram. This is somewhat unavailable to describe the traffic flow characteristic of urban expressway because the metastable state is one typical flow characteristic of urban expressway in China [12, 13]. Therefore, model modification taking into account metastable state is necessary to analysis of the effect of traffic incident on urban expressway.

The objective of this study is to propose a methodology to estimate the impact of traffic incident on urban expressway based on the modified cell transmission model. As the traffic flow characteristic of incident section is entirely different from other sections, it should be separated effectively in order to describe the impact of traffic incidents more accurately. For this purpose, the model should meet a precondition that the length of cell is variable. However, the CEM must divide the cell evenly. Therefore, our study will use the modified CEM which was proposed by Muñoz et al. [8].

The proposed methodology for estimating impact of traffic incident on urban expressway is presented in the next section. Application and evaluation of the proposed methodology are conducted in Section 3. Finally, study summary, directions and issues for further research, and usages of outcome are provided.

\section{Methodology}

2.1. Modified Cell Transmission Model. Modified cell transmission model (MCTM) defines cell densities as state variables instead of cell occupancies and accepts nonuniform cell lengths. By using cell densities instead of cell occupancies, the MCTM is allowed to accommodate uneven cell lengths, which leads to greater flexibility in partitioning highways. In the MCTM, a highway is partitioned into a series of cells. The traffic density in any cell $i$ that evolves according to conservation of vehicles can be expressed as

$$
\rho_{i}(k+1)=\rho_{i}(k)+\frac{\Delta t}{l_{i}}\left(q_{i, \text { in }}(k)-q_{i, \text { out }}(k)\right),
$$

where $\rho_{i}(k+1)$ is the density in cell $i$ during the $(k+1)$ th time interval; $\rho_{i}(k)$ is the density in cell $i$ during the $k$ th time interval; $l_{i}$ is the length of cell $i$; in order to avoid situations that traffic flow value of cell $i$ is negative and the traffic density is greater than the jam density, $l_{i}$ must be longer than the free flow travel distance; $\Delta t$ is the discrete time interval; $q_{i, \text { in }}(k)$ and $q_{i \text { out }}(k)$ are, respectively, the total flows, in vehicles per unit time, entering and leaving cell $i$ during the $k$ th time interval.

The congestion state of cell $i$ is determined by comparing the cell density $\rho_{i}(k)$ with the critical density $\rho_{i, o}(k)$ : if $\rho_{i}(k)<$ $\rho_{i, o}(k)$, the cell $i$ has free flow state during the $k$ th time interval; and if $\rho_{i}(k) \geq \rho_{i, o}(k)$, the cell $i$ is said to have congested state. The inflow to cell $i$ in the time interval $(k, k+$ 1) can be expressed as

$$
y_{i}(k)=q_{i}(k) \Delta t=\min \left[S_{i-1}(k), R_{i}(k)\right],
$$

where $q_{i}(k)$ is the flow entering cell $i$ from the mainline; $S_{i-1}(k)$ is the maximum flow that can be supplied by cell $i-1$ under free flow conditions during the $k$ th time interval; $R_{i}(k)$ is the maximum flow that can be received by cell $i$ under congested conditions over the same time interval. $S_{i-1}(k)$ and $R_{i}(k)$ are given by

$$
\begin{aligned}
S_{i-1}(k) & =\min \left(v_{i-1} \rho_{i-1}(k) \Delta t, Q_{i-1}\right), \\
R_{i}(k) & =\min \left(Q_{i}, w_{i}\left(\rho_{J, i}-\rho_{i}(k)\right) \Delta t\right),
\end{aligned}
$$

where $Q$ is the maximum flow rate; $v$ is the average speed at which vehicles travel down the highway under uncongested (low density) conditions; $w$ is the average speed at which congestion waves propagate upstream within congested (high density) regions of the highway; $\rho_{J, i}$ is the jam density.

Three different types of cell connection are allowed: simple connection, merges, and diverges.

Simple Connection. Figure 1(a) shows the representation of a simple connection. The inflow to cell $i$ in the time interval $(k, k+1)$ can be determined by (2) and (3).

Merges. A merge connection corresponds to the case where more than two links enter and one leaves, as shown in Figure 1(b). In this situation, three cases should be considered.

Case 1. The downstream cell can accept both supply flows from the upstream cell $\left(\sum_{j} S_{i-1, j}(k) \leq R_{i}(k)\right)$. The inflow to cell $i$ in the time interval $(k, k+1)$ can be expressed as

$$
y_{i, \text { in }}(k)=\sum_{j} S_{i-1, j}(k) .
$$

Case 2. Each approach with no priority and the combined supply flow exceed the maximum receiving flow $\left(\sum_{j} S_{i-1, j}(k)>R_{i}(k)\right)$. The inflow from approach $j$ to cell $i$ in the time interval $(k, k+1)$ can be expressed using the following equation:

$$
y_{i, j}(k)=\min \left\{S_{i-1, j}(k), p_{i-1, j}(k) \cdot R_{i}(k)\right\},
$$

where $p_{i-1, j}(k)$ denotes the ratio that the flow from approach $j$ to cell $i$ in the time interval $(k, k+1)$ of the total flow received by cell $i$ over the same time interval, $\sum_{j} p_{i-1, j}(k)=1$. Thus, $y_{i, \text { in }}(k)$ can be mathematically expressed by

$$
y_{i, \text { in }}(k)=\sum_{j} y_{i, j}(k) .
$$

Case 3. The combined supply flows exceed the maximum receiving flow, and vehicles of each approach travel to downstream cell in the order of priority. $y_{i, j}(k)$ can be expressed as

$$
y_{i, j}(k)=\min \left\{S_{i-1, j}(k), R_{i}(k)-\sum_{j^{o}} S_{i-1}^{j^{o}}(k)\right\},
$$




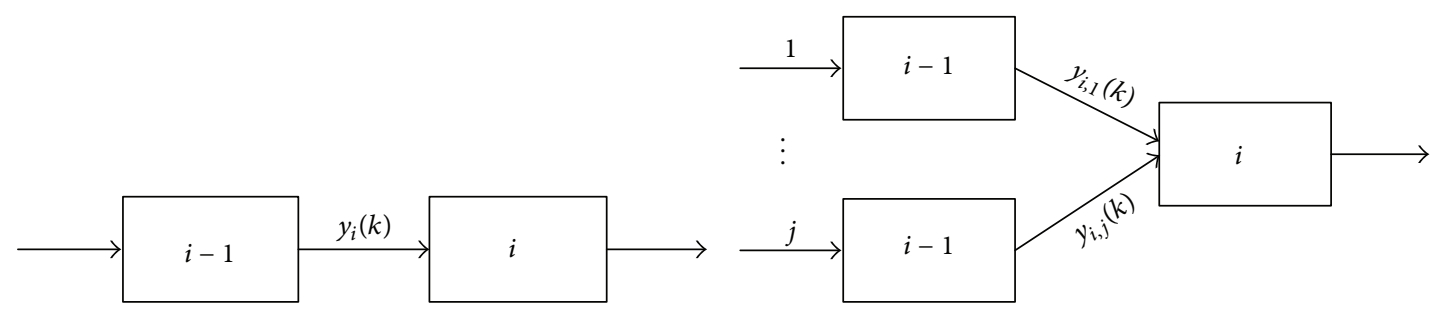

(a) Simple connection

(b) Merges

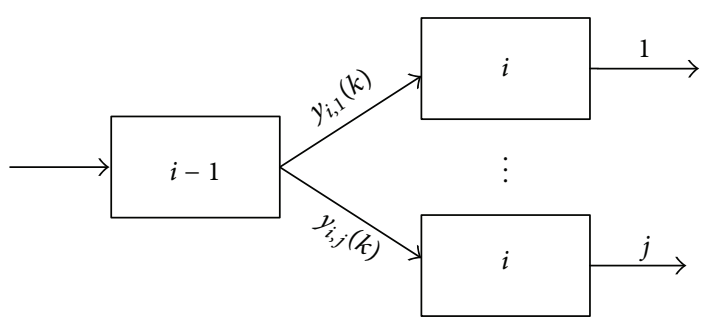

(c) Diverges

FigURE 1: Representation of connection types.

where $\sum_{j^{\circ}} S_{i-1}^{j^{o}}(k)$ denotes the maximum flow supplied by other approaches which take precedence over approach $j$ from cell $i-1$ to cell $i$ in the time interval $(k, k+1)$.

Diverges. If the outflow from a cell is split between the downstream mainline region, one diverges connection is formed, and an example is shown in Figure 1(c):

$$
\begin{aligned}
y_{i, j}(k) & =p_{i}^{j}(k) \cdot y_{i-1, \text { out }}(k), \\
y_{i-1, \text { out }}(k) & =\min \left\{S_{i-1}(k), \frac{R_{i, 1}(k)}{p_{i}^{1}(k)}, \ldots, \frac{R_{i, j}(k)}{p_{i}^{j}(k)}\right\},
\end{aligned}
$$

where $y_{i-1, \text { out }}(k)$ is the total flow leaving cell $i-1$ in the time interval $(k, k+1)$ and $p_{i}^{j}(k)$ is the split ratio.

2.2. Impact of Metastable State. We assume that the ramp of urban expressway does not have signal control, and the flow between any origin-destination pair is constant. In addition, the route used by each driver is effectively fixed in advance, and in particular, drivers do not change their routes in response to congestion. The relationship diagram of flow and density as shown in Figure 2 is adopted to perform the impact of metastable state.

From Figure 2, it is clear that when the cell density equals $\rho_{c}$ or $\rho_{f}$, the cell flow can be estimated based on

$$
\begin{aligned}
\frac{\rho_{I}}{1 / v+1 / w} & =v \rho_{c}, \\
Q & =v \rho_{f},
\end{aligned}
$$

where $\rho_{c}$ is the density from congested state to free flow state and $\rho_{f}$ is the density from free flow state to congested state.

The traffic flow state of cell $i$ is also determined by comparing the cell density $\rho_{i}$ with the critical density: if

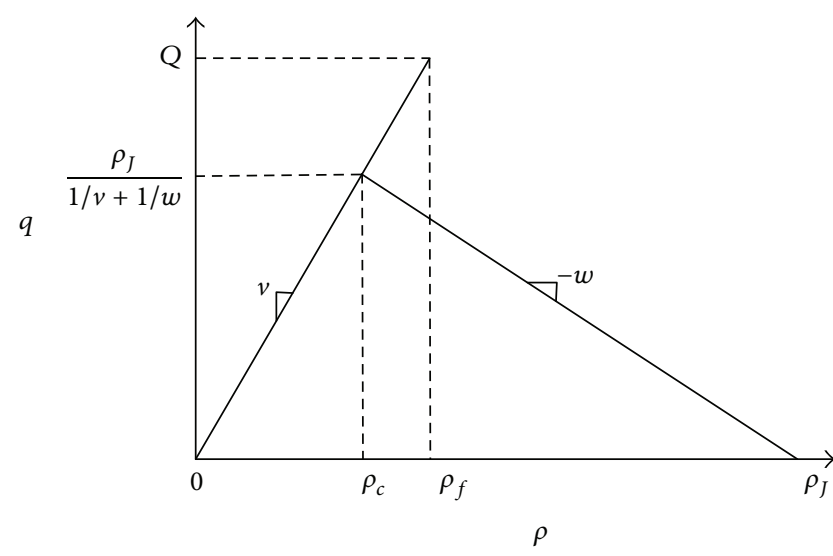

FIGURE 2: Flow-density relationship diagram with metastable state.

$\rho_{i}<\rho_{c}$, the cell $i$ has free flow state, if $\rho_{i}>\rho_{f}$, the cell $i$ has congested state, and if $\rho_{c} \leq \rho_{i} \leq \rho_{f}$, the cell $i$ has metastable state. We define $Z_{i}(k)$ as the traffic state in cell $i$ during the $k$ th time interval. $Z_{i}(k)=0$ represents that the cell $i$ has free flow state during the $k$ th time interval. Similarly, $Z_{i}(k)=1$ represents the congested state, and the metastable state during the $k$ th time interval can be expressed as $Z_{i}(k)=$ $Z_{i}(k-1)$ :

$$
Z_{i}(k)= \begin{cases}0, & \rho \leq \rho_{c}, \\ 1, & \rho \geq \rho_{f}, \\ Z_{i}(k-1), & \rho_{c}<\rho<\rho_{f} .\end{cases}
$$

With these conventions, $S_{i-1}(k)$ and $R_{i}(k)$ in (2) can be rewritten as follows:

$$
\begin{aligned}
S_{i-1}(k) & =\min \left(v_{i-1} \rho_{i-1}(k) \Delta t, Q_{i-1}\right), \\
R_{i}(k) & =\left(1-Z_{i}(k)\right) Q_{i}+Z_{i}(k) w_{i}\left(\rho_{J}-\rho_{i}(k)\right) \Delta t .
\end{aligned}
$$




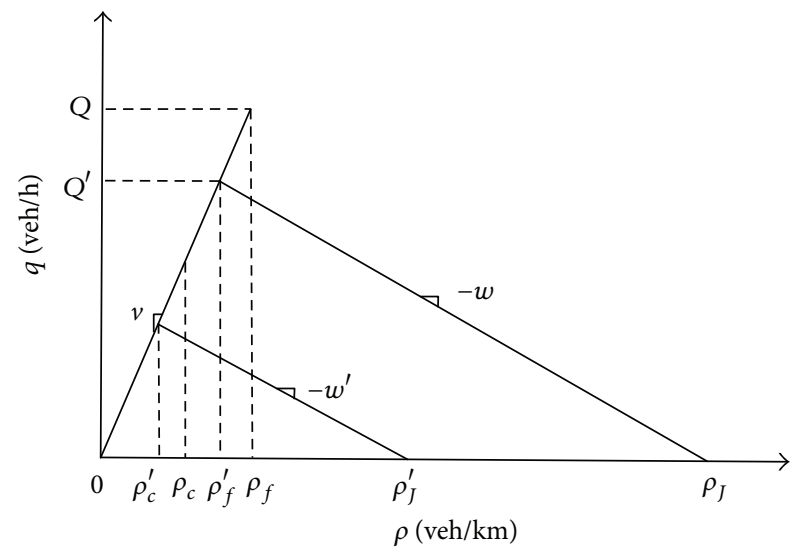

FIGURE 3: Flow-density relationship diagram affected by traffic incidents.

2.3. Impact of Traffic Incident. As the impact of traffic incident, $Q, \rho_{J}, \rho_{c}, \rho_{f}$, and $w$ of controlled cell would be reduced to $Q^{\prime}, \rho_{J}^{\prime}, \rho_{c}^{\prime}, \rho_{f}^{\prime}$, and $w^{\prime}$, as shown in Figure 3. In addition, there are significant differences of traffic flow characteristics between normal operation sections and incident affected sections. Therefore, the normal operation section in the downstream and incident affected section in the upstream should be separated to ensure the position where traffic accident occurred is always at the boundary of cell.

According to the effect of traffic incident occurring in the middle of initial cell, three cases are taken into account to adjust cell initial settings. Note that it is unnecessary to change cell initial settings when traffic incidents occurred in the boundary of cell.

Case 1. The effective length towards to upstream $l_{i}^{\prime}$ and the normal operation length of downstream $l_{i}^{\prime \prime}$ are both shorter than the free flow travel distance $v_{i} \Delta t$. The original cell $i-1$ and road section of $l_{i}^{\prime}$ can reorganize as a new cell $i-1$, the original cell $i+1$, and road section of $l_{i}^{\prime \prime}$ set as a new cell $i$; an example is shown in Figure $4(\mathrm{a})$. Thus $S_{i-1}(k)$ and $R_{i-1}(k)$ can be expressed by (12); meanwhile, the formulas for $S_{i}(k)$ and $R_{i}(k)$ are consistent with (3):

$$
\begin{aligned}
& S_{i-1}(k)=\min \left(v_{i-1}^{\prime} \rho_{i-1}^{\prime}(k) \Delta t, \lambda_{i-1} Q_{i-1}\right), \\
& R_{i-1}(k)=\min \left(\lambda_{i-1} Q_{i-1}, w_{i-1}^{\prime}\left(\rho_{J}^{\prime}-\rho_{i-1}^{\prime}(k) \Delta t\right)\right),
\end{aligned}
$$

where $v_{i-1}^{\prime}$ is the average speed reduced by traffic incident at which vehicles travel down the urban expressway under uncongested (low density) conditions; $w_{i-1}^{\prime}$ is the average speed reduced by traffic incident at which congestion waves propagate upstream within congested (high density) regions of the urban expressway; $\rho_{i-1}^{\prime}(k)$ is the density affected by traffic incident in cell $i-1$ during the $k$ th time interval; $\rho_{J}^{\prime}$ is the jam density affected by traffic incident.

Case 2. $l_{i}^{\prime} \geq v_{i} \Delta t$ and $l_{i}^{\prime \prime}<v_{i} \Delta t$. The road section of $l_{i}^{\prime}$ is regarded as a new cell $i$, a new cell $i+1$ is rebuild by the original cell $i+1$ and road section of $l_{i}^{\prime \prime}$, as shown in Figure 4(b).
The formulas for $S_{i+1}(k)$ and $R_{i+1}(k)$ are consistent with (3). $S_{i}(k)$ and $R_{i}(k)$ would have been

$$
\begin{aligned}
& S_{i}(k)=\min \left(v_{i}^{\prime} \rho_{i}^{\prime}(k) \Delta t, \lambda_{i} Q_{i}\right), \\
& R_{i}(k)=\min \left(\lambda_{i} Q_{i}, w_{i}^{\prime}\left(\rho_{J}^{\prime}-\rho_{i}^{\prime}(k) \Delta t\right)\right) .
\end{aligned}
$$

Case 3. $l_{i}^{\prime}<v_{i} \Delta t$ and $l_{i}^{\prime \prime} \geq v_{i} \Delta t$. The original cell $i-1$ and road section of $l_{i}^{\prime}$ can reorganize as a new cell $i-1$, and the road section of $l_{i}^{\prime \prime}$ is regarded as a new cell $i$ (an example shown in Figure 4(c)). In this case, the formulas for $S_{i}(k)$ and $R_{i}(k)$ are consistent with (3). $S_{i-1}(k)$ and $R_{i-1}(k)$ can calculate by (12).

Thus, such a modified MCET, which specifically considered the metastable phenomenon, would effectively predict the impact of traffic incident on urban expressway. In order to quantify the impact of the traffic incident, it is necessary to determine the evaluation indices. A variety of evaluation indices have been proposed to evaluate the traffic congestion from different perspectives, such as the journey time, travel speed, road running indices, traffic congestion index [14-16], and the area of the traffic jam [1]. However, a few indices are applied to nonrecurrent congestion which is caused by accidents, bad weather, or other random events. In our study, the effected length is put forward to directly depict the influence scope of traffic incident. It can be defined as the total length of congestion cell within the controlled area during the $k$ th time interval. That is,

$$
I L(k)=\sum_{j} l_{j, c}, \quad j \in G,
$$

where $I L(k)$ is the effected length during the $k$ th time interval, $l_{j, c}$ is the length of congestion cell $j$, and $G$ is the controlled area. Note that congestion cell refers to the cell of which density $\rho_{i}$ is greater than the critical density $\rho_{i, o}$.

\section{Application and Evaluation}

In order to apply the methodology introduced in the previous section, the real traffic incident data of urban expressway would be adopted. The comparative analysis of simulation result and real results is constructed to evaluate the practicability of modified MCET. The study section is the segment from Cai HuYing bridge to Fu Cheng Men bridge of west second ring road in Beijing. Total length of the study segment is 6516 meters and the traffic flow direction is northbound.

We have divided the study segment into 20 cells, as shown in Figure 5. The cell index is located in the center of each cell. The uppermost row of numbers above cells gives the number of lanes ( 3 to 4 ) in each cell. The second row of numbers is the cell length (in meter). The location numbers of the mainline loop detectors are listed below the cells. On- and off-ramps are depicted as numbered arrows, and up arrows represent on-ramps, otherwise off-ramps. In our default partitioning method, the locations of on-ramps, off-ramps, and mainline microwave detectors are the cell boundaries. It should be noted that the cell lengths must be longer than the freeflow travel distance (time step of $10 \mathrm{sec}$, the free flow speed as discussed later). If some cells cannot be satisfied with 


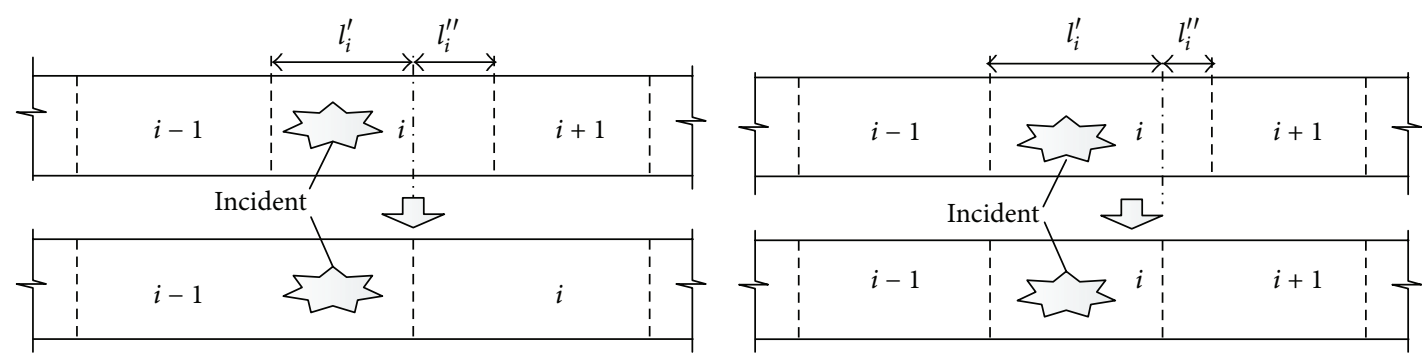

(a) $l_{i}^{\prime}<v_{i} \Delta t$ and $l_{i}^{\prime \prime}<v_{i} \Delta t$

(b) $l_{i}^{\prime} \geq v_{i} \Delta t$ and $l_{i}^{\prime \prime}<v_{i} \Delta t$

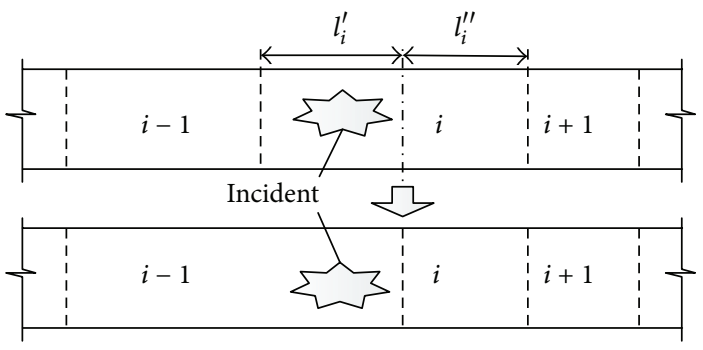

(c) $l_{i}^{\prime}<v_{i} \Delta t$ and $l_{i}^{\prime \prime} \geq v_{i} \Delta t$

FIGURE 4: Cell restructuring settings.

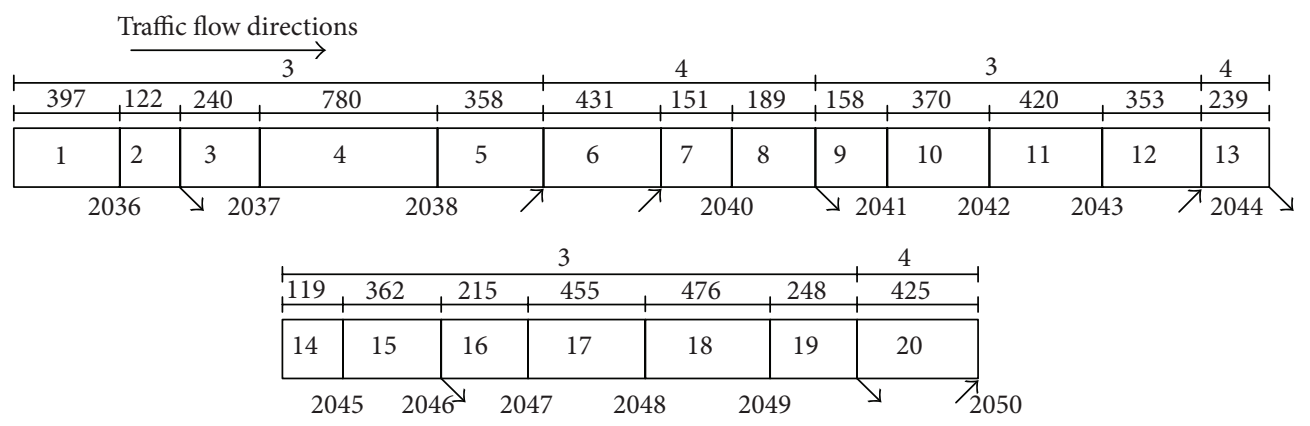

FIgURE 5: Cell partition of the study section.

this condition, we can increase the length of these cells by occupying lengths from their corresponding downstream cells. Alternatively, we can merge these cells with the adjacent cells.

According to the real data, one rear-end accident occurred near Fu Cheng Men bridge at 10:25 on May 29, 2012. The location of this accident is at approximately $300 \mathrm{~m}$ upstream of 2048 detector. One inside lane was occupied. The effected length of this accident is 4548 meter. The accident was cleared away at 11:10 of the same day. The traffic flow returned to normal state until 11:45; in other words, this accident duration is $80 \mathrm{~min}$.

Table 1 shows the contour plots of measured densities from 10:20 to 11:45 am on May 29, 2012. The numbers in bold and italic fonts are traffic densities, in vehicles per mile per lane (vpmpl). Free-flow densities $(0-33 \mathrm{vpmpl})$ are shown in normal font. Midrange congestion (33-43 vpmpl) is in italic font. Bold font indicates heavy congestion ( $43 \mathrm{vpmpl}$ or greater). Traffic is flowing from left to right in this plot, and the time, in 5-minute intervals, is given in the leftmost column.
In this study, we have been working with two main sources of data: microwave detector data obtained from road transport management system (RTMS) and a set of manually counted volumes of urban expressway. The RTMS-derived data used in this study includes the flow (veh/h), speed, and occupancy rate at each detector, which is available in 5 min and consecutive of $24 \mathrm{~h}$. However, the RTMS data is always missed due to the malfunctioning detectors. Therefore, the manually counted data is recorded to supplement the data set. We can substitute a historical average of the manually counted flows for that missing data. Our procedure for estimating the MCTM split ratios is to compute, for each offramp, the ratio of the measured off-ramp flow to the total measured flow exiting the diverge junction. Since we have access to only a limited amount of accurate off-ramp data, we are currently using historically averaged split ratios in our modified MCTM simulations.

To estimate the impact of the traffic accident using the modified MCET, the MCTM parameters should be calibrated. The calibration procedure of free flow speed $v$, the jam density $\rho_{J, i}$, the shock speed $w$, nonbottleneck capacity, and 
TABLE 1: Contour plots for measured densities from 10:20 a.m. to 11:45 a.m. on May 29, 2012.

\begin{tabular}{ccccccccccccccccc}
\hline & 2036 & 2037 & 2038 & 2039 & 2040 & 2041 & 2042 & 2043 & 2044 & 2045 & 2046 & 2047 & 2048 & 2049 & 2050 \\
\hline $10: 20$ & 23 & 24 & 27 & 29 & 28 & 28 & 23 & 27 & 25 & 24 & 28 & 29 & 25 & 21 & 26 \\
$10: 25$ & 22 & 25 & 26 & 29 & 29 & 27 & 25 & 28 & 28 & 27 & 50 & 65 & 42 & 21 & 26 \\
$10: 30$ & 25 & 26 & 25 & 28 & 27 & 30 & 28 & 27 & 42 & 49 & $\mathbf{6 7}$ & $\mathbf{7 6}$ & 43 & 23 & 26 \\
$10: 35$ & 22 & 25 & 26 & 32 & 29 & 29 & 27 & 37 & 56 & 56 & $\mathbf{6 7}$ & $\mathbf{8 3}$ & 47 & 23 & 29 \\
$10: 40$ & 24 & 27 & 33 & 29 & 29 & 28 & 38 & 44 & $\mathbf{7 3}$ & $\mathbf{6 7}$ & $\mathbf{7 6}$ & $\mathbf{8 1}$ & 39 & 23 & 28 \\
$10: 45$ & 22 & 26 & 27 & 29 & 28 & 34 & 47 & 61 & $\mathbf{6 9}$ & $\mathbf{6 8}$ & $\mathbf{7 7}$ & $\mathbf{8 4}$ & 38 & 22 & 25 \\
$10: 50$ & 21 & 26 & 31 & 28 & 29 & 42 & 61 & $\mathbf{6 8}$ & $\mathbf{7 4}$ & $\mathbf{7 1}$ & $\mathbf{7 9}$ & $\mathbf{9 0}$ & 36 & 23 & 26 \\
$10: 55$ & 26 & 25 & 28 & 54 & 60 & $\mathbf{6 8}$ & $\mathbf{7 2}$ & $\mathbf{7 4}$ & $\mathbf{7 5}$ & $\mathbf{7 3}$ & $\mathbf{8 1}$ & $\mathbf{9 4}$ & 42 & 23 & 29 \\
$11: 00$ & 25 & 37 & 39 & 53 & 61 & 65 & 64 & $\mathbf{8 8}$ & $\mathbf{7 9}$ & $\mathbf{7 7}$ & $\mathbf{8 1}$ & $\mathbf{9 5}$ & 38 & 22 & 25 \\
$11: 05$ & 25 & 29 & 47 & 54 & 44 & $\mathbf{7 2}$ & $\mathbf{6 6}$ & $\mathbf{8 9}$ & $\mathbf{9 2}$ & $\mathbf{8 8}$ & $\mathbf{9 7}$ & $\mathbf{9 5}$ & 35 & 26 & 27 \\
$11: 10$ & 28 & 32 & 59 & $\mathbf{6 8}$ & 42 & 54 & $\mathbf{6 7}$ & $\mathbf{7 3}$ & $\mathbf{8 5}$ & $\mathbf{8 1}$ & $\mathbf{8 6}$ & 65 & 29 & 26 & 25 \\
$11: 15$ & 28 & 28 & 29 & 42 & 42 & 42 & 50 & 64 & $\mathbf{6 9}$ & 64 & 59 & 56 & 40 & 35 & 27 \\
$11: 20$ & 29 & 27 & 36 & 33 & 34 & 43 & 42 & 48 & 64 & 56 & 58 & 46 & 44 & 34 & 28 \\
$11: 25$ & 22 & 27 & 29 & 29 & 28 & 33 & 43 & 42 & 43 & 42 & 48 & 43 & 42 & 28 & 26 \\
$11: 30$ & 25 & 26 & 26 & 31 & 28 & 29 & 29 & 41 & 37 & 37 & 43 & 40 & 41 & 31 & 26 \\
$11: 35$ & 25 & 26 & 25 & 33 & 27 & 27 & 32 & 28 & 35 & 28 & 35 & 38 & 40 & 30 & 25 \\
$11: 40$ & 24 & 25 & 24 & 27 & 29 & 29 & 27 & 29 & 29 & 27 & 34 & 27 & 35 & 29 & 26 \\
$11: 45$ & 25 & 24 & 26 & 29 & 28 & 28 & 26 & 29 & 30 & 24 & 28 & 27 & 37 & 30 & 27 \\
$11: 50$ & 25 & 28 & 27 & 26 & 26 & 25 & 22 & 27 & 28 & 21 & 24 & 28 & 27 & 27 & 26 \\
\hline
\end{tabular}
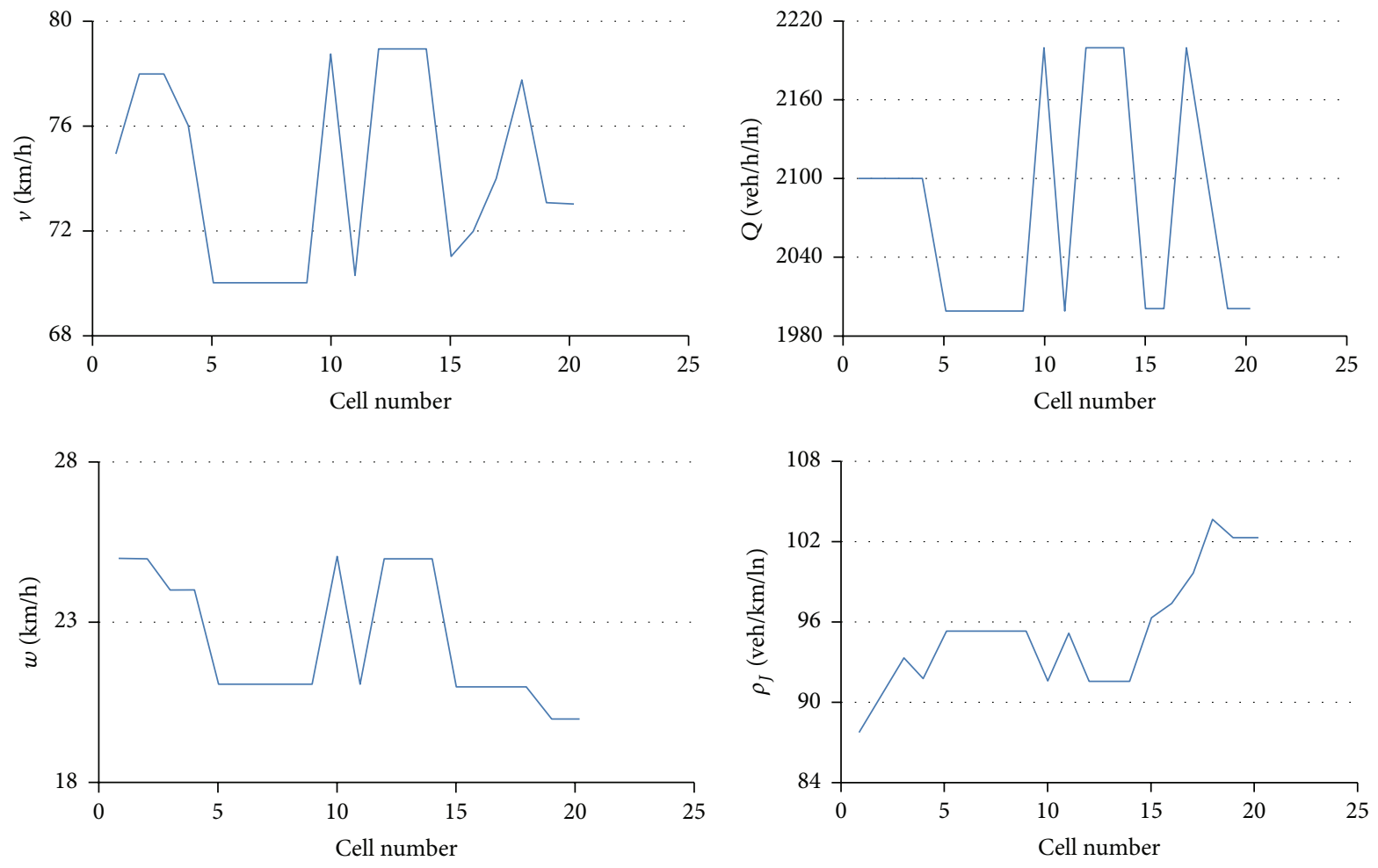

FIGURE 6: Calibration of modified MCTM parameters.

bottleneck capacity has been presented systematically in the literature [8]. On the basis of this calibration method and the real data, the range of these parameters is obtained. Free flow speed $v$ is typically in the range of $80-90 \mathrm{~km} / \mathrm{h}$, nonbottleneck capacity of each lane is $1700-2200 \mathrm{veh} / \mathrm{h}$, shock speed $w$ is
$20-30 \mathrm{~km} / \mathrm{h}$, and jam density $\rho_{J, i}$ is $235-285 \mathrm{veh} / \mathrm{km}$. The calibration results of these parameters are shown in Figure 6.

According to cell partition of the study section, the accident placed in the middle of 17 th cell. The effective length towards upstream $l_{17}^{\prime}$ is 155 meter, and the normal operation 


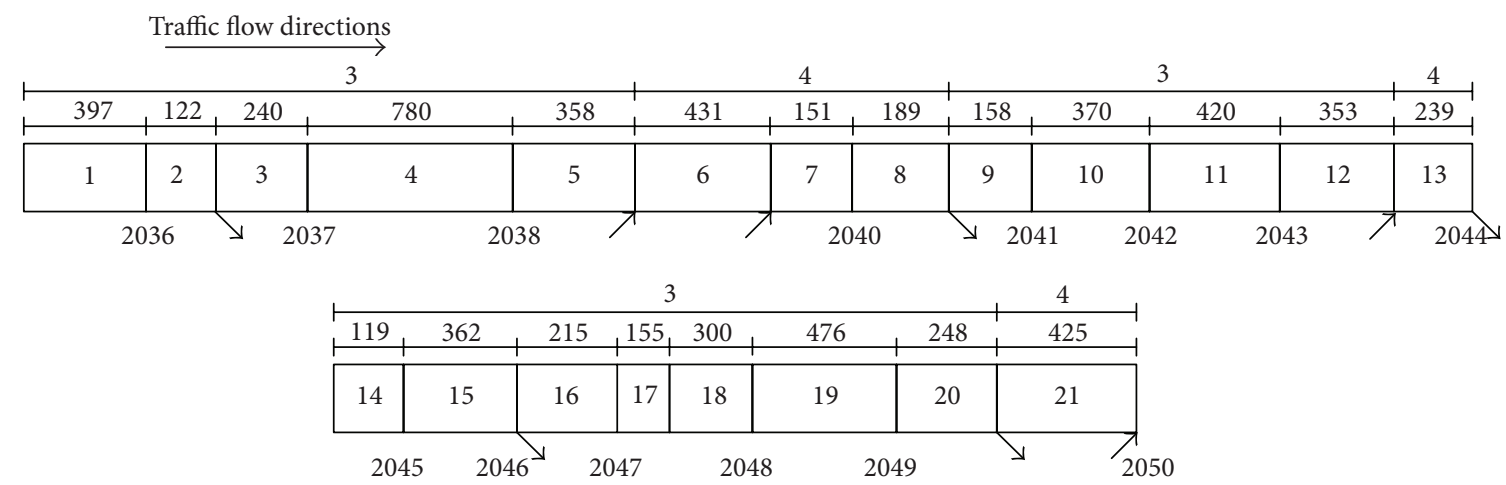

FIGURE 7: Cell restructuring settings of the study section.

TABLE 2: Contour plots for simulated densities from 10:20 a.m. to 11:45 a.m. on May 29, 2012.

\begin{tabular}{lccccccccccccccc}
\hline & 2036 & 2037 & 2038 & 2039 & 2040 & 2041 & 2042 & 2043 & 2044 & 2045 & 2046 & 2047 & 2048 & 2049 & 2050 \\
\hline $10: 20$ & 21 & 20 & 23 & 26 & 26 & 25 & 21 & 26 & 24 & 22 & 28 & 28 & 24 & 20 & 25 \\
$10: 25$ & 21 & 22 & 25 & 26 & 27 & 27 & 22 & 27 & 26 & 26 & 29 & 35 & 37 & 21 & 25 \\
$10: 30$ & 21 & 22 & 25 & 27 & 27 & 28 & 24 & 29 & 28 & 43 & 55 & $\mathbf{8 5}$ & 38 & 21 & 26 \\
$10: 35$ & 22 & 24 & 27 & 28 & 27 & 29 & 29 & 29 & 28 & 62 & 77 & $\mathbf{8 5}$ & 38 & 22 & 26 \\
$10: 40$ & 22 & 24 & 27 & 28 & 28 & 28 & 28 & 46 & 47 & $\mathbf{7 0}$ & $\mathbf{7 7}$ & $\mathbf{8 5}$ & 38 & 23 & 26 \\
$10: 45$ & 22 & 26 & 27 & 28 & 28 & 28 & 28 & 51 & 54 & 71 & $\mathbf{8 1}$ & $\mathbf{8 8}$ & 39 & 23 & 26 \\
$10: 50$ & 23 & 27 & 27 & 28 & 29 & 27 & 40 & 62 & $\mathbf{7 5}$ & $\mathbf{7 2}$ & $\mathbf{8 1}$ & $\mathbf{8 9}$ & 39 & 23 & 27 \\
$10: 55$ & 25 & 27 & 28 & 29 & 63 & 47 & 61 & $\mathbf{7 5}$ & $\mathbf{7 6}$ & $\mathbf{7 9}$ & $\mathbf{8 4}$ & $\mathbf{9 1}$ & 40 & 23 & 27 \\
$11: 00$ & 25 & 28 & 28 & 29 & 60 & 61 & 75 & $\mathbf{7 5}$ & $\mathbf{8 1}$ & $\mathbf{7 9}$ & $\mathbf{8 8}$ & $\mathbf{9 5}$ & 43 & 24 & 27 \\
$11: 05$ & 26 & 28 & 49 & 39 & 54 & $\mathbf{8 4}$ & $\mathbf{7 5}$ & $\mathbf{7 9}$ & $\mathbf{8 1}$ & $\mathbf{8 2}$ & $\mathbf{9 0}$ & $\mathbf{9 9}$ & 43 & 24 & 27 \\
$11: 10$ & 26 & 39 & 43 & 56 & 44 & 62 & 57 & $\mathbf{7 9}$ & $\mathbf{7 7}$ & $\mathbf{7 3}$ & $\mathbf{7 5}$ & 61 & 40 & 25 & 27 \\
$11: 15$ & 25 & 37 & 40 & 48 & 32 & 53 & 50 & 63 & $\mathbf{7 3}$ & $\mathbf{7 1}$ & 60 & 51 & 40 & 43 & 28 \\
$11: 20$ & 24 & 28 & 35 & 41 & 31 & 46 & 46 & 51 & 65 & 63 & 58 & 42 & 39 & 41 & 28 \\
$11: 25$ & 24 & 26 & 29 & 37 & 29 & 34 & 42 & 45 & 57 & 56 & 43 & 42 & 38 & 41 & 28 \\
$11: 30$ & 24 & 26 & 25 & 31 & 28 & 30 & 34 & 34 & 47 & 41 & 43 & 38 & 37 & 40 & 28 \\
$11: 35$ & 23 & 24 & 25 & 27 & 28 & 27 & 25 & 29 & 36 & 37 & 35 & 35 & 37 & 39 & 27 \\
$11: 40$ & 23 & 22 & 24 & 27 & 26 & 27 & 23 & 28 & 23 & 25 & 28 & 32 & 35 & 39 & 27 \\
$11: 45$ & 22 & 21 & 23 & 26 & 25 & 26 & 23 & 26 & 21 & 23 & 26 & 28 & 26 & 37 & 26 \\
$11: 50$ & 22 & 21 & 23 & 24 & 25 & 26 & 21 & 25 & 26 & 21 & 25 & 26 & 24 & 35 & 26 \\
\hline
\end{tabular}

length of downstream $l_{17}^{\prime \prime}$ equals 300 meter. As the free flow travel distance $v_{i} \Delta t$ is 114 meter, the conditions that $l_{17}^{\prime} \geq v \Delta t$ and $l_{17}^{\prime \prime} \geq v \Delta t$ are obviously satisfied. Therefore, these two partial sections can be separated to become two new cells, as shown in Figure 7.

In addition, the characteristic traffic flow parameters of 17 th cell have reduced due to the impact of accident. Figure 8 shows the traffic-density relationship diagram of 17 th cell. It revealed that the value of $Q, \rho_{J}, \rho_{c}, \rho_{f}$, and $w$ at normal operating state is $6300 \mathrm{veh} / \mathrm{h} / \mathrm{ln}, 276 \mathrm{veh} / \mathrm{km} / \mathrm{ln}$, $63 \mathrm{veh} / \mathrm{km} / \mathrm{ln}, 89 \mathrm{veh} / \mathrm{km} / \mathrm{ln}$, and $21 \mathrm{~km} / \mathrm{h}$, respectively. As the accident, the value of these parameters changed into $Q^{\prime}$, $\rho_{J}^{\prime}, \rho_{c}^{\prime}, \rho_{f}^{\prime}$, and $w^{\prime}$, which, respectively, equals $3087 \mathrm{veh} / \mathrm{h} / \mathrm{ln}$, $138 \mathrm{veh} / \mathrm{km} / \mathrm{ln}, 31 \mathrm{veh} / \mathrm{km} / \mathrm{ln}, 44 \mathrm{veh} / \mathrm{km} / \mathrm{ln}$, and $20.48 \mathrm{~km} / \mathrm{h}$.

The modified MCTM put forward in the previous section was used to simulate this accident. In order to reduce the random error of simulation, the average result of 10 times simulation is regarded as the final simulation results.
The contour plot for simulated density is shown in Table 2. From Tables 1 and 2, it can be seen that the contour plot for measured and simulated density has almost the same trends. And the measured and simulated diffusion range of congestion all spreads to the location 2036 and 2037 detector. Compared with actual duration, the simulation duration is $85 \mathrm{~min}$, which predicts with approximately $6.25 \%$ error. Furthermore, the simulated result of effected length is 4670 meter, and the error is $2.68 \%$. These errors are within an acceptable range.

\section{Conclusion}

Unlike traditional approaches that use cell transmission model to measure the impact of traffic incident for freeway, this study proposed a methodology to predict the impact of traffic incident on urban expressway using the modified MCET. In order to reflect the actual operating state of 


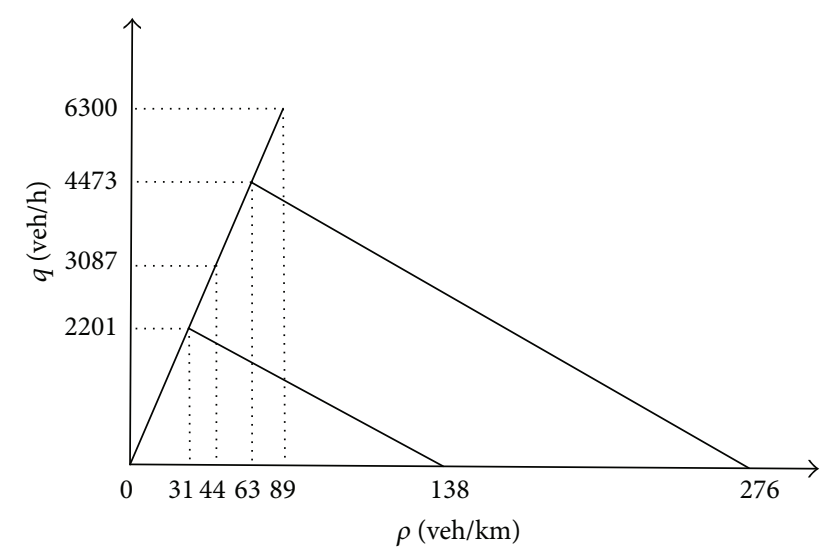

FIGURE 8: Traffic-density relationship diagram of accident section.

traffic flow on urban expressway as much as possible, the metastable state was simulated in this modified MCET. In the meantime, the main traffic flow parameters of incident cell were adjusted on the basis of the impact of traffic incidents. With regard to the location of traffic incidents, the method of cell restructuring settings was discussed. Additionally, one evaluation indicator which denotes the effected length is put forward to directly depict the influence scope of traffic incident. To fully apply the proposed methodology, a simulation is developed to evaluate whether the methodology is available or not. This analysis is based on the data microwave detector obtained road transport management system (RTMS) and set of manually counted volumes of urban expressway. The simulation results have revealed that the proposed methodology can accurately predict the impact of traffic incidents on urban expressway.

Although the availability of the proposed prediction method is demonstrated, further research should be conducted to fully implement this methodology in the field. Also, more significant analysis on the impact of metastable state is required. The methodology in this study is useful to recognize the influence of traffic incident on urban expressway accurately, which can be further utilized in intelligent traffic control and as information for control strategies of urban expressway. In addition, opportunities exist for deriving effective countermeasures with traffic incidents for improving the traffic efficiency of urban expressway.

\section{Conflict of Interests}

The authors declare that there is no conflict of interests regarding the publication of this paper.

\section{Acknowledgment}

This work was supported by the National Natural Science Foundation of China (no. 51478110).

\section{References}

[1] C. Wright and P. Roberg, "The conceptual structure of traffic jams," Transport Policy, vol. 5, no. 1, pp. 23-35, 1998.
[2] A. Skabardonis, P. Varaiya, and K. F. Petty, "Measuring recurrent and nonrecurrent traffic congestion," Transportation Research Record: Journal of the Transportation Research Board, no. 1856, pp. 118-124, 2003.

[3] J. Kwon and P. Varaiya, "The congestion pie: delay from collisions, potential ramp metering gain, and excess demand," in Proceedings of the 84th Annual Meeting of the Transportation Research Board, Washington, DC, USA, 2005.

[4] J. C. Long, Z. Y. Gao, H. L. Ren, and A. P. Lian, "Urban traffic congestion propagation and bottleneck identification," Science in China, Series F: Information Sciences, vol. 51, no. 7, pp. 948964, 2008.

[5] R. Smid, The variability of traffic in congestion forecasting [M.S. thesis], Delft University of Technology, Delft, The Netherlands, 2012.

[6] C. F. Daganzo, “The cell transmission model: a dynamic representation of highway traffic consistent with the hydrodynamic theory," Transportation Research Part B: Methodological, vol. 28, no. 4, pp. 269-287, 1994.

[7] C. F. Daganzo, “The cell transmission model, part II: network traffic," Transportation Research Part B, vol. 29, no. 2, pp. 79-93, 1995.

[8] L. Muñoz, X. Sun, R. Horowitz, and L. Alvarez, "Traffic density estimation with the cell transmission model," in Proceedings of the American Control Conference, vol. 5, pp. 3750-3755, IEEE, June 2003.

[9] W. Y. Szeto, B. Ghosh, B. Basu, and M. O’Mahony, "Multivariate traffic forecasting technique using cell transmission model and SARIMA model," Journal of Transportation Engineering, vol. 135, no. 9, pp. 658-667, 2009.

[10] J. Long, Z. Gao, X. Zhao, A. Lian, and P. Orenstein, "Urban traffic jam simulation based on the cell transmission model," Networks and Spatial Economics, vol. 11, no. 1, pp. 43-64, 2011.

[11] A. Sumalee, R. X. Zhong, T. L. Pan, and W. Y. Szeto, "Stochastic cell transmission model (SCTM): a stochastic dynamic traffic model for traffic state surveillance and assignment," Transportation Research Part B: Methodological, vol. 45, no. 3, pp. 507-533, 2011.

[12] X. Sun, H. Lu, and J. Wu, "Research on speed-flow-density relationship model of Beijing urban expressway," Highway Engineering, no. 1, pp. 43-48, 2012.

[13] S. Wu, X. Zheng, M. Guo, and Z. Wu, "Survey report on urban freeway traffic flow of several typical cities in China," Scientia Sinica (Physica, Mechanica \& Astronomica), no. 6, pp. 791-800, 2011.

[14] T. Lomax, S. Turner, G. Shunk et al., Quantifying Congestion. Volume 1: Final Report, Transportation Research Board, 1997.

[15] M. G. Boarnet, E. J. Kim, and E. Parkany, "Measuring traffic congestion," Transportation Research Record, no. 1634, pp. 9399, 1998.

[16] H. K. Lo, "A novel traffic signal control formulation," Transportation Research Part A: Policy and Practice, vol. 33, no. 6, pp. 433-448, 1999. 


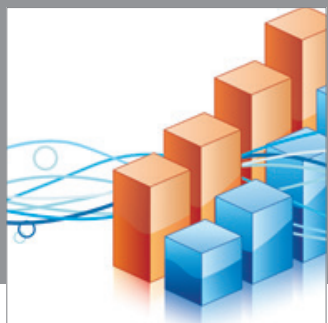

Advances in

Operations Research

mansans

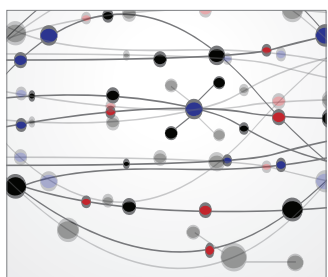

The Scientific World Journal
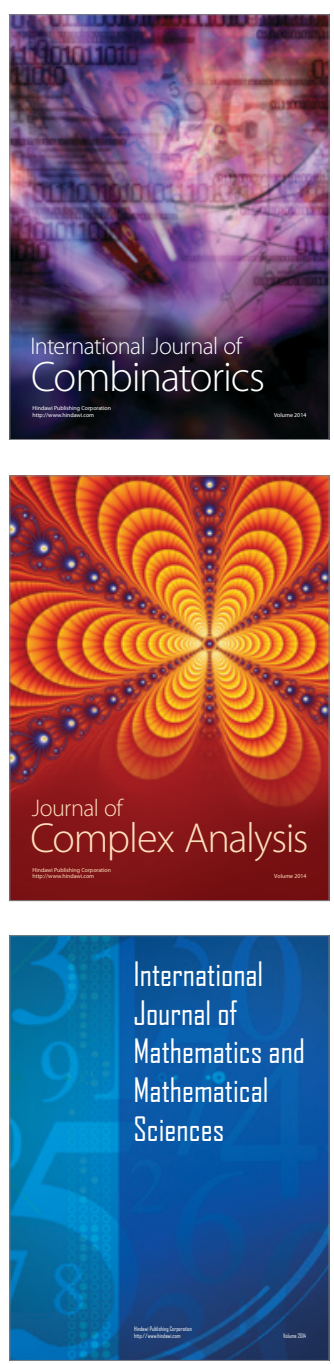
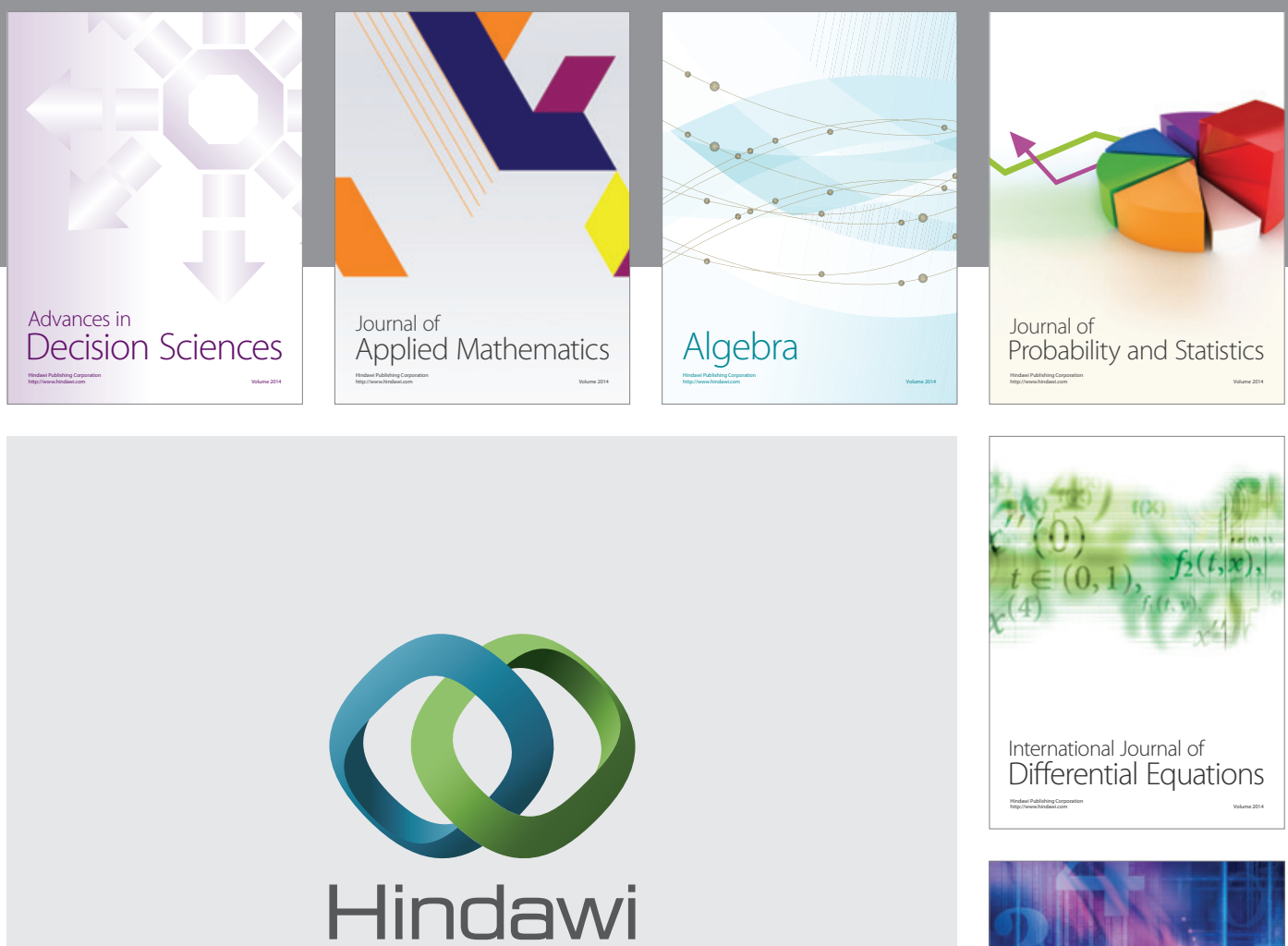

Submit your manuscripts at http://www.hindawi.com
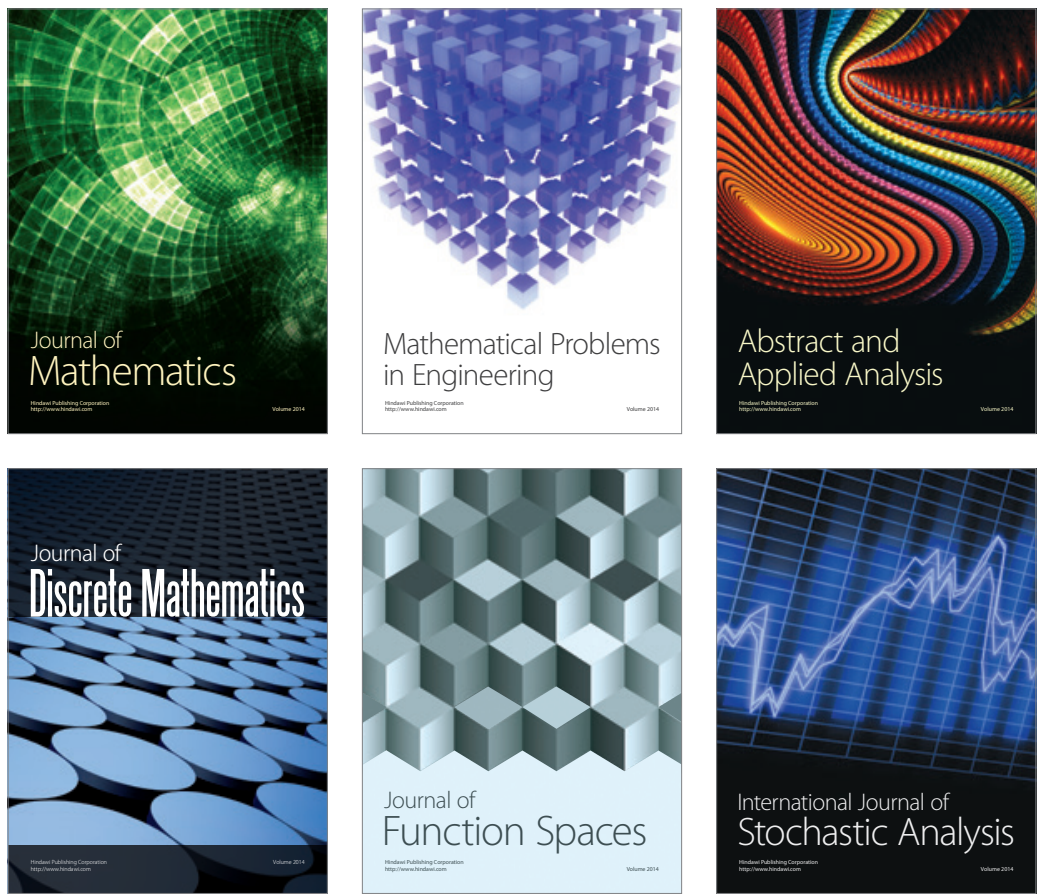

Journal of

Function Spaces

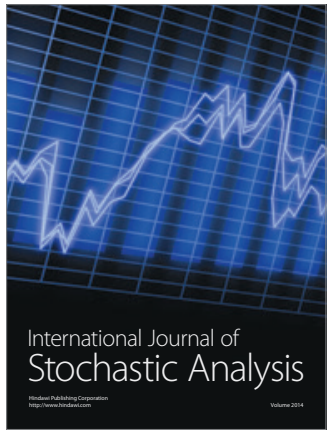

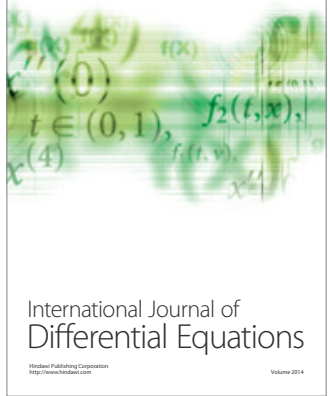
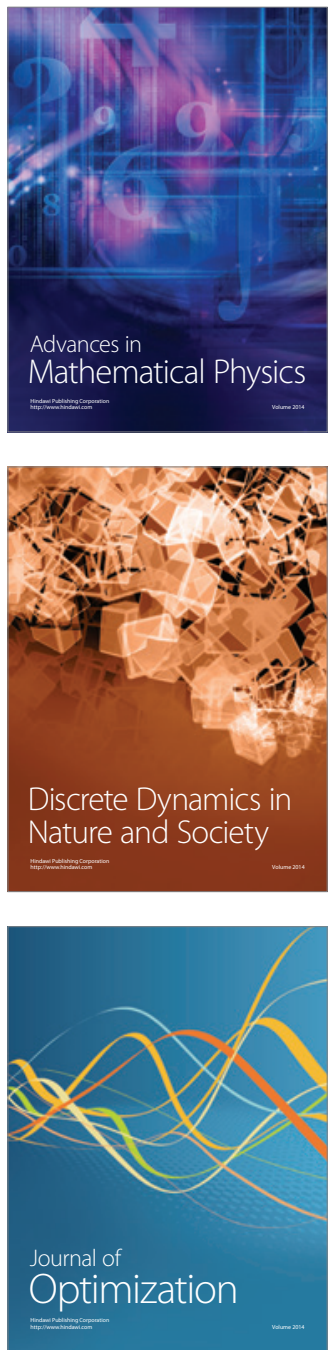